Société d'histoire de la révolution de 1848 et des

révolutions du XIXe siècle

$30 \mid 2005$

Pour une histoire culturelle de la guerre au XIXe siècle

\title{
Evelyn Blewer, Secours mutuel. Victor Hugo et la crise des théâtres parisiens, 1848-1849, Saint-Pierre du Mont, Eurédit, 2002, 361 p.
}

Judith Lyon-Caen

\section{(2) OpenEdition}

Édition électronique

URL : http://journals.openedition.org/rh19/1053

DOI : $10.4000 /$ rh 19.1053

ISSN : $1777-5329$

Éditeur

La Société de 1848

Édition imprimée

Date de publication : 1 juin 2005

ISSN : 1265-1354

Référence électronique

Judith Lyon-Caen, « Evelyn Blewer, Secours mutuel. Victor Hugo et la crise des théâtres parisiens,

1848-1849, Saint-Pierre du Mont, Eurédit, 2002, 361 p. », Revue d'histoire du XIXe siècle [En ligne], 30

2005, mis en ligne le 19 février 2006, consulté le 10 décembre 2020. URL : http://

journals.openedition.org/rh19/1053; DOI : https://doi.org/10.4000/rh19.1053

Ce document a été généré automatiquement le 10 décembre 2020.

Tous droits réservés 


\title{
Evelyn Blewer, Secours mutuel. Victor Hugo et la crise des théâtres parisiens, 1848-1849, Saint-Pierre du Mont, Eurédit, 2002, 361 p.
}

\author{
Judith Lyon-Caen
}

Secours mutuel aborde à la fois un chapitre rarement étudié de la carrière de Victor Hugo et un aspect peu fouillé de la période de la Révolution de 1848 : Hugo joua en effet un rôle de premier plan dans les multiples tentatives de sauvetage du monde des théâtres parisiens, profondément affectés par la révolution de Février. Ce livre présente deux parties bien distinctes : c'est tout d'abord une chronique de l'activité de l'écrivain au service d'un monde en crise, qui se clôt en juillet 1849 quand la Chambre refuse de renouveler les secours aux théâtres accordés l'été précédent, alors que Hugo oriente sa vie parlementaire dans d'autres directions (la défense de la presse, l'éducation, la misère populaire). Secours mutuel présente ensuite un important dossier de lettres inédites, émanant de Hugo ou de personnages du monde des théâtres et provenant de fonds multiples (Archives Nationales, Bibliothèque Nationale, Maison de Victor Hugo, archives de la Société des auteurs et compositeurs dramatiques et archives de la Fondation Taylor). Grande connaisseuse des papiers de Victor Hugo, Evelyn Blewer, qui a notamment collaboré à l'édition de la Correspondance familiale ${ }^{1}$, a ainsi pu réunir 77 lettres, qui sont ici très soigneusement éditées et annotées.

2 La révolution de Février se traduisit par un effondrement de la fréquentation des salles de théâtre parisiennes: alors que Frédérick Lemaître reprenait triomphalement des représentations gratuites du Chiffonnier de Paris de Félix Pyat au Théâtre de la Porte Saint-Martin en ajoutant une couronne dans sa hotte de chiffonnier, les recettes de l'ensemble des théâtres parisiens chutèrent en quelques semaines. Ainsi le même Théâtre de la Porte Saint-Martin justement, qui faisait 65000 francs de recettes en janvier 1848, voit-il ses recettes tomber à 18000 francs en mai, et à 6500 francs en juillet, alors qu'il faisait plus de 40000 francs en juillet 1847 - l'été rimant toujours avec 
un repli de la fréquentation des salles. Le Théâtre-Français, rebaptisé Théâtre de la République, connaît une évolution similaire ; l'Ambigu-Comique, comme d'autres, finit par fermer ses portes. Le public déserte donc les salles de théâtre, qui ont de lourdes charges à payer, dont l'impôt dit du " onzième ", ou droit des indigents, établi en 1699 et qui servait à financer les hospices : il était calculé quotidiennement sur la recette brute des théâtres et était perçu aux caisses par les agents de l'administration des hospices. En cas de baisse des recettes, le onzième grevait lourdement les marges bénéficiaires des théâtres, dont les frais fixes étaient élevés, notamment à cause de la multiplication des spectacles et de la surenchère de décors coûteux.

Le Gouvernement provisoire, fort proche du monde des lettres, fut d'emblée sensible à la situation dramatique des salles. Les directeurs de théâtres, menés par Hippolyte Hostein, obtinrent en mars la création d'une Commission nationale des théâtres, composée d'un délégué du Gouvernement, de quatre représentants des directeurs de théâtres, d'un représentant des acteurs, de trois journalistes et de trois auteurs dramatiques. Cette Commission devait être le lieu d'élaboration d'une véritable politique culturelle, puisqu'elle avait en charge non seulement la question du «droit des hospices ", mais encore celles des privilèges, des subventions et de la censure. Les artistes dramatiques y étaient fort mal représentés, et s'en inquiétèrent : l'Association des artistes dramatiques, fondée en 1840 par le baron Taylor pour assurer une pension aux acteurs âgés et venir aux secours des plus démunis, se réunit bien vite pour exiger une plus large représentation au sein de la Commission, et tenta de s'allier dans ce but avec la Société des auteurs et compositeurs dramatiques, eux aussi mal représentés, et dont les relations avec les directeurs de théâtres étaient également naturellement tendues, puisque bien des entrepreneurs devaient de l'argent aux auteurs comme aux acteurs. La Commission obtint en mars 1848 une réduction d'impôts: de fait, la situation des théâtres était si mauvaise que l'acquittement de ces droits pourtant amoindris fut parfois inexistant, au même titre que le versement des droits d'auteurs.

En cette période préélectorale, alors que les travaux de la Commission des théâtres sont suspendus, la Société des auteurs et compositeurs dramatiques tente de se doter d'un représentant sur la scène politique nationale : Victor Hugo entre en scène. L'ancien pair de France n'est officiellement pas candidat aux élections du 23 avril, mais il ne refuse pas absolument de l'être. Sous la pression, il devient le favori d'un ensemble d'associations "professionnelles» d'artistes menées par Taylor (peintres, musiciens, ouvriers et inventeurs industriels) et se présente comme le candidat en qui se reconnaîtront «les écrivains, les poètes, les artistes, les hommes de la pensée », cette " autre classe d'ouvriers », celle des «travailleurs de l'intelligence». Trop peu soutenu par la grande presse, Hugo n'est pas élu ; mais il a trouvé dans le désarroi des artistes un tremplin politique. Devenu président de la Société des auteurs et compositeurs dramatiques en mai 1848, il la dote d'une vision et d'une stratégie politiques: aux hommes de plume, qui ont largement accès à la publicité, il convient de savoir faire pression sur l'Assemblée constituante pour influer sur l'élaboration de la législation théâtrale. De nouveau candidat en mai, avec plus de conviction et de moyens publicitaires que le mois précédent, Hugo est élu aux élections complémentaires de juin dans le département de la Seine : ce n'est pas seulement un représentant du peuple qui entre alors à la Chambre, mais un représentant du monde des théâtres, dont l'élection doit beaucoup aux réunions, aux affiches, aux tracts du "Club des lettres et des arts", du nom de la fédération d'associations d'artistes que vient de former le baron Taylor. 
5 La situation des théâtres, au mois de juin, devient catastrophique : la Commission nationale se réunit à nouveau le 7 juin, s'ouvrant cette fois aux représentants des Auteurs et compositeurs dramatiques. Hugo y intervient longuement pour défendre la liberté des entreprises théâtrales mais surtout pour promouvoir « un grand théâtre normal », une " magnifique exploitation théâtrale » d'État, «représentant toutes les formes de l'art, pouvant payer avec splendeur poètes et artistes », concentrant donc tous les moyens, et se disputant la «faveur publique » avec les autres théâtres, qui ne seraient pas écrasés mais poussés à l'excellence par cette concurrence démesurée. L'heure n'est pourtant pas aux grands projets, et c'est la question du secours immédiat aux théâtres qui est au cœur des débats de l'été 1848. Evelyn Blewer décrit ici avec une grande précision l'ensemble des démarches des multiples acteurs de cette affaire, ainsi que le débat parlementaire qui aboutit à débloquer 680000 francs pour l'Opéra et les théâtres de Paris le 17 juillet 1848.

6 L'activité théâtrale reprit un peu, mais de nombreux théâtres et de nombreuses catégories d'artistes s'estimaient lésés par les dispositions du décret du 17 juillet. Hugo, qui reçoit de nombreuses lettres de sollicitations d'artistes au chômage, obtient de la Constituante le vote de 300000 francs «d'encouragement " aux beaux-arts et aux belles-lettres. En réalité, la situation des théatres redevient rapidement critique: l'actualité politique (élection présidentielle en décembre 1848, élections législatives en mai) concurrence les distractions théâtrales; l'épidémie de choléra qui se déclare en mars 1849 incite à éviter les lieux publics; et, comme le note Evelyn Blewer, il est possible que l'image même des théâtres ait souffert de la quête aux subventions du printemps et de l'été 1848. Hugo refait campagne pour les élections de juin sans le soutien des associations théâtrales : les clubs étant interdits et le cautionnement de la presse politique rétabli, ni les associations ni les journaux de théâtre ne se risquent dans la politique. De leur côté, les directeurs de théâtres se constituent de nouveau en lobby pour obtenir de l'argent. Au mois de juillet, Hugo se bat pour obtenir le renouvellement des secours de l'année passée. La demande de crédit est finalement repoussée par la Chambre, qui note que la situation des théâtres est malheureusement celle «d'une foule d'autres industries qui ne sont ni moins compromises, ni moins respectables » : «Faire une exception en faveur des théatres ne serait pas de la justice ni d'une bonne administration des ressources de l'État». Pour utiliser des termes actuels, l'argument de « l'exception culturelle » est donc repoussé.

7 Ainsi s'achève un épisode qui vit revenir Hugo au cœur de la scène politique nationale et ouvrit un important débat sur les relations de l'État et des établissements culturels dans un pays où la subvention d'une partie des activités artistiques est très ancienne. C'est ici que l'on peut regretter le caractère trop étroitement monographique du travail d'Evelyn Blewer, en dépit de la richesse et de la qualité de son information. La chronique linéaire ne permet pas de cartographier clairement les opinions et les intérêts qui s'affrontent dans cette affaire, y compris les positions de Victor Hugo. Aucune des questions soulevées en 1848 n'est par ailleurs véritablement neuve, et il aurait pu être intéressant d'en évoquer la généalogie, la postérité et les inflexions. Enfin, l'affaire de la crise des théâtres parisiens en 1848-1849 croise deux autres phénomènes, qui ne sont évoqués ici qu'allusivement : d'une part, l'année 1848 est le moment d'une intense réflexion sur le rôle de la littérature et des arts dans une révolution, qui aboutit, on le sait, à un retrait de nombre d'artistes et d'écrivains de la scène politique. Dumas, comme Hugo, se présente aux élections législatives d'avril 1848 
en candidat des ouvriers de la pensée et en soulignant la dette de la Révolution à l'égard du monde des lettres; Proudhon repousse violemment cette candidature, au nom de l'inutilité absolue, voire de la nocivité, de la littérature. D'autre part, cette crise des théâtres fait apparaître l'intensité des mouvements de regroupement qu'ont connus les métiers des arts et des lettres sous la Monarchie de Juillet ; elle incite ainsi à une réflexion plus ample sur les lieux, les moments et les modalités de l'autonomisation des «professions» artistiques et du «travail intellectuel» dont l'histoire n'a été jusqu'ici qu'ébauchée.

\section{NOTES}

1.. Correspondance familiale et écrits intimes, sous la direction de Jean GAUDON, Sheïla GAUDON et Bernard LEUILLOT, Paris, Éditions Robert Laffont, collection «Bouquins », 1991 et 1998, 2 tomes. 